\title{
Efecto de la gonadotrofina coriónica equina sobre el porcentaje de preñez y pérdidas embrionarias en vacas Braford
}

\author{
Errico, S. ${ }^{3}$; Insaugarat, J. ${ }^{2}$; Errico, R. ${ }^{2}$; Uslenghi, G. ${ }^{1,4}$; Callejas, S.S. ${ }^{1}$ \\ 'Área Reproducción, FISFARVET. Centro Investig. Vet. Tandil (CIVETAN, CONICET-CICPBA), Fac.Cs.Vet., \\ Univ. Nac. Centro Prov. Buenos Aires, Argentina. Tel.: 0249-4385850. ${ }^{2}$ Actividad privada, 25 de Mayo, \\ Argentina. ${ }^{3}$ Tesinista Fac.Cs.Vet. ${ }^{4}$ Becario CONICET. E-mail: callejas@vet.unicen.edu.ar
}

\begin{abstract}
Resumen
Errico, S.; Insaugarat, J.; Errico, R.; Uslenghi, G.; Callejas, S.S.: Efecto de la gonadotrofina coriónica equina sobre el porcentaje de preñez y pérdidas embrionarias en vacas Braford. Rev. vet. 27: 2, 121-123, 2016. El objetivo de este trabajo fue evaluar el efecto de la administración de gonadotrofina coriónica equina (eCG) en el momento de retirar un dispositivo intravaginal con progesterona (DISP) y/o 16 días posteriores a la inseminación artificial a tiempo fijo (IATF), sobre el porcentaje de preñez y la mortalidad embrionaria entre los días 30 y 55 post IATF en vacas Braford en pobre condición corporal (1,5 a 2, escala 1 a 5). Éstas ( $\mathrm{n}=162)$ fueron distribuidas aleatoriamente a 4 grupos, grupo eCG0: El día -8 se colocó un DISP con $750 \mathrm{mg}$ de progesterona más una inyección de $2 \mathrm{mg}$ de benzoato de estradiol. El día 0 se retiró el DISP y se administraron $0,150 \mathrm{mg}$ de cloprostenol sódico, $1 \mathrm{mg}$ de cipionato de estradiol y $400 \mathrm{UI}$ de eCG. Grupo eCG16: ídem tratamiento anterior excepto que las 400 UI de eCG se administraron el día 16. Grupo eCG0y16: ídem tratamiento eCG0, administrando además 400 UI de eCG en el día 16. Grupo control: ídem grupo eCG0 sin administrar eCG. La IATF se realizó a las 48-52 $\mathrm{h}$ post retiro del DISP. El diagnóstico de gestación se realizó a las 30 y 55 días post IATF. La administración de eCG en el día del retiro del DISP mejoró el porcentaje de preñez en los días $30(49,4 \%$ vs. $28,8 \%)$ y $55(48,1 \%$ vs. $26,3 \%)$ post IATF ( $<<0,05)$, independientemente si se utilizó o no eCG en el día 16. El porcentaje de mortalidad embrionaria no fue influenciado por la eCG. En conclusión, la administración de eCG en el momento de retirar un DISP en vacas con cría con pobre condición corporal (1,5 a 2) mejora el porcentaje de preñez a la IATF. El uso de la eCG en el día 16 post retiro del dispositivo no afecta la preñez. Las pérdidas embrionarias no se modifican al utilizar eCG.
\end{abstract}

Palabras clave: vaca, reproducción, eCG, IATF, condición corporal, preñez.

\begin{abstract}
Errico, S.; Insaugarat, J.; Errico, R.; Uslenghi, G.; Callejas, S.S.: Effect of equine chorionic gonadotropin on pregnancy rate and embryo lost rate in Braford cows after artificial insemination. Rev. vet. 27: 2, 121-123, 2016. The objective of the present study was to evaluate the effect of equine chorionic gonadotropin $(\mathrm{eCG})$ at withdrawal of a progesterone intravaginal device (PID) and/or 16 days after fixed-time artificial insemination (FTAI) on pregnancy rate and embryo lost rate between days 30 and 55 after FTAI on suckled Braford beef cows of low corporal condition $(1.5-2$, scale $1-5)$. Cows $(n=162)$ were randomly distributed to four treatments; group eCG0: on day -8 cows received a PID with $750 \mathrm{mg}$ of progesterone (P4) plus 2 $\mathrm{mg}$ of estradiol benzoate (EB). On day 0 PID were removed and cows received $0.150 \mathrm{mg}$ of cloprostenol, $1 \mathrm{mg}$ of estradiol cypionate (ECP) and $400 \mathrm{IU}$ of eCG. Group eCG16: cows received the same treatments than group eCG0, but eCG was administered on day 16. Group eCG0-16: cows received the same treatments than group eCG0, plus another injection of eCG on day 16 . Control group: cows received the same treatments than group eCG0, without eCG. FTAI was performed 48-52 h after PID removal. Pregnancy diagnosis was performed by ultrasonography on days 30 and 55 after FTAI. The administration of eCG at PID removal enhanced pregnancy rate on days $30(49.4 \%$ vs. $28.8 \%)$ and $55(48.1 \%$ vs. $26.3 \%)(\mathrm{p}<0.05)$ and have no effect on pregnancy loses. In conclusion, administration of eCG at the moment of PID removal in suckled beef cows of low corporal condition (1.5-2) increased pregnancy rate at FTAI. Administration of eCG 16 days after FTAI do not affect pregnancy rate; eCG do not affect pregnancy loses.
\end{abstract}

Key words: cow, reproduction, eCG, FTAI, corporal condition, pregnancy. 


\section{INTRODUCCIÓN}

A fines de la década de 1990 y principios del año 2000, la ganadería comenzó a ser desplazada por la agricultura, siendo la soja el principal competidor debido a su alta rentabilidad. En consecuencia, los campos de mejor calidad son hoy destinados a agricultura, desplazando a la ganadería hacia tierras de menor potencial. La actividad más afectada fue la cría, a la cual se le asignaron recursos forrajeros de menor calidad, además de un aumento de la carga animal, afectándose los índices reproductivos de los rodeos.

Nutricionalmente afectadas, las vacas actualmente llegan a la temporada de servicio en una pobre condición corporal, hecho que se agrava año tras año. Si a ello se le suma el drenaje por amamantamiento, se genera un balance energético negativo que incrementa el período de anestro post parto, afectando notablemente el sistema productivo ${ }^{9}$.

Por otro lado, con la finalidad de mejorar la eficiencia reproductiva y productiva del sistema, se ha implementado el uso de la inseminación artificial a tiempo fijo (IATF). Este sistema controla el ciclo estral de los animales mediante el uso combinado de dispositivos intravaginales con progesterona, estrógenos y un agente luteolítico ${ }^{1}$.

En las vacas con pobre condición corporal está afectada la frecuencia de pulsos de LH y el desarrollo folicular, perjudicándose la ovulación -en cantidad y calidad- y la tasa de preñez $3,8,9,11$, incrementándose las pérdidas por mortalidad embrionaria ${ }^{8}$.

Este cuadro justifica el uso de gonadotrofina coriónica equina (eCG) con la finalidad de estimular el desarrollo folicular, administrándola en el momento de retirar los dispositivos intravaginales ${ }^{9}$. Por otro lado, recientemente han surgido publicaciones que preconizan la administración de eCG luego de realizar una IATF, con la finalidad de disminuir el porcentaje de hembras con mortalidad embrionaria ${ }^{6}$, por lo cual resulta de interés profundizar los conocimientos sobre el efecto de la eCG en la eficiencia reproductiva, administrándola durante el tratamiento de inducción de la ovulación para IATF y/o posteriormente a la misma.

El objetivo del presente trabajo fue evaluar el efecto de la administración de eCG en el momento de retirar un dispositivo intravaginal y/o 16 días posteriores de la IATF sobre el porcentaje de preñez en vacas con cría que sobrellevan una pobre condición corporal. Además se estudió el efecto que esta hormona ejerce sobre las pérdidas embrionarias/fetales entre los días 30 y 55 post IATF.

\section{MATERIAL Y MÉTODOS}

Lugar, animales y alimentación. El ensayo se realizó en un establecimiento mixto ubicado en el Partido de 25 de Mayo, Provincia de Buenos Aires. Se utilizaron 162 vacas Braford con cría al pie, con una sanidad óptima y una condición corporal promedio de $2,3 \pm 0,8$ (escala de 1 a 5). La alimentación se basó en campo natural de baja calidad.

Revisación ginecológica. Los ovarios fueron revisados por ultrasonografía (transductor transrectal de $10 \mathrm{MHz}$, Easyscan) determinando la estructura ovárica predominante (EOP) al momento de comenzar con los tratamientos de control del ciclo estral (folículos menores a $10 \mathrm{~mm}$; folículos mayores o iguales a $10 \mathrm{~mm}$ y cuerpo lúteo, como señala la Tabla 1).

Tabla 1. Estructura ovárica predominante (EOP) en vacas al iniciar el tratamiento de control de ciclo estral.

\begin{tabular}{lcc}
\hline EOP & $\mathrm{n}$ & $\%$ \\
\hline folículos menores & 48 & 29,6 \\
folículos mayores & 96 & 59,2 \\
cuerpo lúteo & 18 & 11,2 \\
\hline
\end{tabular}

n: número de vacas (total: 162); \%: porcentaje

Tratamientos. Las vacas fueron distribuidas de forma aleatoria, teniendo en cuenta la EOP, según un diseño factorial $2 \times 2$ a 4 grupos para recibir los siguientes tratamientos (efectos principales: momento de aplicación de la eCG, en el día 0 y en el día 16):

- Grupo eCG-0 (n=40): el día -8 se colocó un dispositivo intravaginal con $750 \mathrm{mg}$ de progesterona micronizada (Pro-Ciclar P4 Zoovet, Lab.Zoovet) más una inyección de $2 \mathrm{mg}$ de benzoato de estradiol (Benzoato de estradiol Zoovet, Lab.Zoovet). El día 0 se retiró el dispositivo y se administró $0,150 \mathrm{mg}$ de cloprostenol sódico (Ciclar ${ }^{\circledR}$, Lab.Zoovet), $1 \mathrm{mg}$ de cipionato de estradiol (Cipionatozoovet y Lab.Zoovet) y 400 UI de eCG (Novormon 5000, Lab.Sintex).

-Grupo eCG-16 (n=41): ídem tratamiento anterior con la salvedad que no se administró eCG en el día 0 y se administró 400 UI de eCG en el día 16 (14 días posteriores a la IATF).

-Grupo eCG-0y16 (n=40): ídem tratamiento eCG-0, administrando además 400 UI de eCG en el día 16.

-Grupo Control ( $n=41)$ : ídem Grupo eCG-0 con la salvedad que no se administró eCG.

Servicio. Se realizó por IATF a las 48-52 h de retirados los dispositivos, utilizando semen congelado/ descongelado en pajuelas de $0,25 \mathrm{ml}$ proveniente de un toro de fertilidad probada.

Diagnóstico de gestación. Se efectuó a los 30 y 55 días post IATF mediante ultrasonografía. La primera ecografía permitió determinar el porcentaje de preñez a la IATF y la segunda estudiar la presencia de mortalidad embrionaria.

Análisis estadístico. Se estudió el efecto de la aplicación de eCG al retiro del dispositivo (día 0 ), en el día 16 y la interacción de ambos sobre el porcentaje de preñez a la IATF en los días 30 y 55. Para realizar el análisis estadístico se utilizó el ProcCATMOD del paquete estadístico SAS ${ }^{10}$, fijando un nivel de confianza del $95 \%(\alpha=0,05)$. 


\section{RESULTADOS Y DISCUSIÓN}

La administración de eCG en el día del retiro del dispositivo mejoró significativamente el porcentaje de preñez en los días 30 y 55 post IATF ( $<<0,05$, Tabla 2 ), independientemente si se utilizó o no eCG en el día 16 (interacción día 0 de administración de eCG $\mathrm{x}$ día 16 : $\mathrm{p}>0,05$ ).

Tabla 2. Tasa de preñez según momento de aplicación de eCG luego de retirado un dispositivo intravaginal con progesterona en vacas con cría IATF (día 0: retiro del dispositivo).

\begin{tabular}{lcc}
\hline efectos principales & \multicolumn{2}{c}{ porcentaje de preñez } \\
\hline adm. eCG en día 0 & día 30 & día 55 \\
SI & $49,4^{\mathrm{a}}(40 / 81)$ & $48,1^{\mathrm{a}}(39 / 81)$ \\
NO & $28,8^{\mathrm{b}}(23 / 80)$ & $26,3^{\mathrm{b}}(21 / 80)$ \\
adm. eCG en día 16 & & \\
SI & $38,8(31 / 80)$ & $37,5(30 / 80)$ \\
NO & $39,5(32 / 81)$ & $37,0(30 / 81)$ \\
\hline
\end{tabular}

adm: administración; ${ }^{\text {a,b }}$ : valores diferentes dentro de cada efecto principal difieren con significancia $\mathrm{p}<0,05$.

La mejoría observada en el porcentaje de preñez en los animales que recibieron eCG, fue coincidente con resultados de otros investigadores ${ }^{6}$, quienes registraron un $62,1 \%$ de preñez para los animales que recibieron eCG y de $48,3 \%$ para los no tratados. En el mismo sentido, se han informado valores de $65,2 \%$ y $34,8 \%$ según recibieron o no $\mathrm{eCG}^{7}$.

El mayor porcentaje de preñez en las vacas que recibieron eCG podría deberse a que esta hormona aumenta la tasa de ovulación y el nivel de progesterona generado por el cuerpo lúteo subsiguiente ${ }^{5}$. No obstante los resultados registrados en el presente trabajo discrepan con los informados por otros autores, quienes no encontraron efecto del tratamiento hormonal ${ }^{4}$. Esto podría deberse a que la zona donde se realizó la IATF, fue afectada por una gran sequía desde diciembre a marzo, por lo cual los animales (con baja condición corporal) no tuvieron posibilidades de disponer de forraje para recuperar la misma.

La administración de eCG en el día 16 no tuvo el efecto esperado (mejorar la tasa de preñez por disminución de la mortalidad embrionaria), discrepando con resultados publicados previamente ${ }^{2,6,7}$. Futuros trabajos deberán clarificar las discrepancias observadas. El porcentaje de mortalidad embrionaria no fue influenciado por el momento de administración de eCG ( $\mathrm{p}>0,05$; $4,8 \%$ ). La interacción entre el día 0 y 16 de aplicada la eCG no fue significativa ( $p>0,05)$.

En conclusión, la administración de eCG en el momento de retirar un dispositivo intravaginal con progesterona en vacas con cría con pobre condición corporal (1,5 a 2) mejora el porcentaje de preñez a la IATF. Contrariamente, el uso de la eCG en el día 16 post retiro del dispositivo no tiene efecto sobre dicho porcentaje. Con respecto a las pérdidas embrionarias, éstas no se mo- difican al utilizar eCG ya sea en el momento de retirar el dispositivo intravaginal, 16 días después o en ambos momentos.

\section{REFERENCIAS}

1. Bó GA, Baruselli PS, Moreno D, Cutaia L, Caccia M, Tríbulo R. 2002. The control of follicular wave development for self-appointed embryo transfer programs in cattle. Theriogenology 57: 53-72.

2. Bó G, Cutaia L, Bartolomé J. 2011. Uso de la eCG asociado a programas reproductivos en vacas lecheras. Anales IX Simposio Internacional de Reproducción Animal, Córdoba (Argentina), p. 139-144.

3. Lamb GC, Stevenson JS, Kesler DJ, Garverick HA, Brown DR, Salfen BE. 2001. Inclusion of a progesterone intravaginal insert plus GnRH and prostaglandin $\mathrm{F}_{2} \alpha$ for ovulation control in postpartum suckled beef cows. $J$ Anim Sci 79: 2253-2259.

4. Maraña PD, Cutaia L, Borges LF, Piancinato D, Perez LC, Rizzi C, Balla E, Bó G. 2005. Efecto de la aplicación de eCG y destete temporario sobre los porcentajes de preñez en vacas posparto tratadas con DIB y benzoato de estradiol. Anales VI Simposio Internacional de Reproducción Animal, Córdoba (Argentina), p. 408.

5. Marques MO, Baruselli PS, Reis EL, Nasser LF, Bó GA. 2004. The use of treatments to improve reproductive performance of anestrus beef cattle tropical climates. Anim Reprod Sci 82/83: 479-486.

6. Menchaca A, Núñez R, Wijma R, García PC, Fabini F, de Castro T. 2013. Como mejorar la fertilidad de los tratamientos de IATF en vacas Bos Taurus. Anales X Simposio Internacional de Reproducción Animal, Córdoba (Argentina), p. 103-133.

7. Núñez R, Castro D, García PC, Bó G, Menchaca A. 2011. Respuesta ovulatoria y luteal luego de una dosis de eCG al finalizar un tratamiento para IATF en vacas de carne en anestro. Anales IX Simposio Internacional de Reproducción Animal, Córdoba (Argentina), p. 339.

8. Perry GA, Smith MF, Lucy MC, Green JA, Parks TE, MacNeil MD, Roberts AJ, Geary TW. 2005. Relationship between follicle size at insemination and pregnancy success. Proc Natl Acad Sci USA 102: 5268-5273.

9. Sá Filho OG, Meneghetti M, Peres RF, Lamb GC, Vasconcelos JL. 2009. Fixed-time artificial insemination with estradiol and progesterone for Bos indicus cows II: Strategies and factors affecting fertility. Theriogenology 72: 210-218.

10. SAS Institute. 1998. SAS/STAT ${ }^{\circledR}$ User's Guide, Version 6, vol. 2, 4th ed., Cary (USA), p. 846.

11. Vasconcelos JL, Sartori R, Oliveira HN, Guenther JG, Wiltbank MC. 2001. Reduction in size of the ovulatory follicle reduces subsequent luteal size and pregnancy rate. Theriogenology 56: 307-314. 